\title{
Abstract Multiplication Semigroups
}

\author{
J.M.A.M. van Neerven \\ Centre for Mathematics and Computer Science \\ P.O. Box 4079, 1009 AB Amsterdam, The Netherlands
}

\begin{abstract}
In this paper we characterise the linear operators on a Banach lattice which are generators of multiplication semigroups. The main result is as follows: Suppose $A$ is a positive band preserving operator with $D(A)$ a Riesz subspace which generates a dense ideal. Then $-A$ admits a unique extension to a multiplication semigroup. If $D(A)$ is a dense ideal, then this extension is the closure. We use this to prove the following: if $A$ is a linear operator on $E$ whose domain generates a dense ideal, then $A$ generates a multiplication semigroup if and only if there is a real $\lambda \in \varrho(A)$ such that $R(\lambda, A)=(\lambda-A)^{-1}$ is positive and band preserving. We also study the duality theory of multiplication semigroups.
\end{abstract}

1980 Mathematics Subject Classification: 47D05, 47B55, 46B30

Keywords \& phrases: multiplication semigroup, band preserving operator, sun-reflexive, atomic Banach lattice.

\section{Introduction}

A $C_{0}$-semigroup $\{T(t)\}_{t \geqslant 0}$ (briefly, $\left.T(t)\right)$ of bounded linear operators on a Banach lattice $E$ will be called a multiplication semigroup if each operator $T(t)$ is a band preserving operator. Multiplication semigroups arise naturally as the Fourier transforms of certain semigroups generated by differential operators. Also, by the spectral theorem, semigroups generated by selfadjoint operators are similar to multiplication semigroups. In this paper we are concerned with the problem to characterise linear operators that generate multiplication semigroups. The starting point are the following results:

(i) If $A$ is the generator of a $C_{0}$-semigroup $T(t)$ on a $\sigma$-Dedekind complete Banach lattice $E$, then $T(t)$ is a multiplication semigroup if and only if $D(A)$ is an ideal and $A x \in B_{x}$ for all $x \in D(A)$ [N2, Thm. C.II.5.13]. Since a bounded band preserving operator preserves ideals, this is in turn equivalent to: $D(A)$ is an ideal and $A x \in \overline{E_{x}}$ for all $x \in D(A)$. Here $B_{x}$ and $E_{x}$ denote the band resp. ideal generated by $x$ in $E$.

(ii) Let $E$ be a Banach functiuon space. The operator $A$, defined as multiplication with a measurable function $g$, generates a multiplication semigroup if and only if $D(A)$ is dense and maximal and $g$ is bounded from above [NP]. The hypotheses imply that $D(A)$ is an ideal; moreover every Banach function space is $\sigma$-Dedekind complete.

In Section 1 we will prove the following generalization of these results. Call an operator $A$ positive if $A x \geqslant 0$ for all $0 \leqslant x \in D(A)$. An operator $A$ with domain $D(A)$ is said to be band preserving if $A x \in B_{x}$ for all $x \in D(A)$.

Theorem 0.1. Suppose $A$ is a positive operator on a Banach lattice $E$. If $D(A)$ is a dense ideal and $A$ preserves bands, then $A$ is closable and $-\bar{A}$ generates a multiplication semigroup. 
Conversely, if $A$ generates a multiplication semigroup, then $D(A)$ is a dense ideal, $A$ preserves closed ideals, and there is a $\lambda \in \mathbb{R}$ such that $\lambda-A$ is positive.

In particular no $\sigma$-Dedekind completeness assumption is made. Examples of non- $\sigma$ Dedekind complete spaces are $C[0,1]$ and $C_{0}(\mathbb{R})$. The generators of multiplication semigroups turn out to be precisely the maximal elements of a certain class of operators to be defined below (Theorem 1.5). This is analogous to the correspondence in Hilbert space between being a generator of a contraction semigroup and being maximally dissipative.

If one is only interested in a unique extension of $A$ that generates a multiplication semigroup, then the first part of Theorem 0.1 may be improved as follows.

Theorem 0.2. Suppose $A$ is a positive band preserving operator with $D(A)$ a Riesz subspace which generates a dense ideal. Then $-A$ admits a unique extension to a generator of a multiplication semigroup.

This theorem is proved in Section 2. An almost immediate consequence is the following extension result: suppose $F \subset E$ is a closed sublattice generating a dense ideal. If $T \in \mathcal{L}(F)$ is a band preserving operator on $F$, then there is a unique extension to a band preserving operator on $E$.

Combination of Theorems 0.1 and 0.2 leads to the following alternative characterization of generators of multiplication semigroups.

Theorem 0.3. Let $A$ be a linear operator on $E$ whose domain generates a dense ideal. Then $A$ generates a multiplication semigroup if and only if there is a real $\lambda \in \varrho(A)$ such that $R(\lambda, A)=(\lambda-A)^{-1}$ is positive and band preserving.

In Section 3 we show that essentially there exist only two classes of Banach lattices which can be $\odot$-reflexive with respect to a multiplication semigroup, viz. the reflexive and the atomic ones.

All vector spaces will be real. Most results have an analogue in the complex case however, cf. the remark after 2.13. We will follow the terminology of the books $[\mathrm{AB}],[\mathrm{M}],[\mathrm{P}]$ and $[\mathrm{N} 2]$.

\section{Proof of Theorem 0.1}

Let $E$ be a Riesz space. We will say that $F$ is a Riesz subspace of $E$ if it is a linear subspace on $E$ with the property that $|x| \in F$ whenever $x \in F$. An operator $A: F \rightarrow E$ between two Riesz spaces $E$ and $F$ is said to be disjointness preserving if $x \perp y$ in $F$ implies $A x \perp A y$ in $E$. A linear operator $T: F \rightarrow E$ is order bounded if it maps order bounded subsets of $F$ into order bounded subsets of $E$. Positive operators are order bounded. A linear operator $A$ on $E$ with domain $D(A)$ a Riesz subspace is called order bounded if $A$ is order bounded as an operator from the Riesz space $D(A)$ to $E$. Finally, a linear operator $A$ on $E$ with domain $D(A)$ is called band preserving if for all $x \in D(A)$ we have $A x \in B_{x}$, the band generated in $E$ by $x$. We start with a lemma which describe some elementary properties of band preserving operators.

Lemma 1.1. (i) Let $A$ be a positive band preserving operator on $E$ with $D(A)$ a Riesz subspace of $E$. If $x \perp y$ in $D(A)$, then $A x \perp A y$ in $E$.

(ii) If $A: F \rightarrow E$ is a disjointness preserving and order bounded map between the Archimeadean Riesz spaces $F$ and $E$, then $|A|$ exists and satisfies $|A x|=|A||x|=|A| x|| . ;$ (i) If $x, y \in D(A)$ such that $|x| \wedge|y|=: z>0$ holds in $E$, then $z \in D(A)$ since $D(A)$ is a sublattice, and consequently $|x| \wedge|y|=z>0$ also holds in $D(A)$. Therefore, if $x \perp y$ in $D(A)$, then $x \perp y$ in $E$ and (i) follows since $A$ preserves bands. The proof of (ii) can be found in [AB, Thm. 8.6]. 
In particular, by (i) and (ii) an order bounded band preserving operator $A$ whose domain is a Riesz subspace is the difference $A^{+}-A^{-}$of two positive band preserving operators. If in addition $D(A)=E$, then $A$ is bounded.

Lemma 1.2. For a linear operator $A$ on a Banach lattice $E$ with $D(A)=E$ the following properties are equivalent:

(a) $A$ is order bounded and band preserving;

(b) $A$ is band preserving;

(c) $A$ is ideal preserving;

(d) there exists a number $\lambda>0$ such that $A \in[-\lambda I, \lambda I]$.

The proof is given in [AB, Ch. 8 and 15].

If $A$ generates a multiplication semigroup $T(t)$, then each $T(t)$ preserves ideals and consequently $A$ perserves closed ideals.

Lemma 1.3. Let $T(t)$ be a multiplication semigroup on a Banach lattice $E$. Then there exists a $\omega \in \mathbb{R}$ such that $0 \leqslant T(t) \leqslant e^{\omega t}$ for all $t \geqslant 0$.

Proof: First we show that $T(t)$ is positive. Since the band preserving operators form a commutative subalgebra of $\mathcal{L}(E)$, which both as Riesz space and as algebra is isomorphic to a space $C(K)$ [AB, Thm 15.5], [M, Thm 3.1.10], we have $T(t)=T(t / 2)^{2} \geqslant 0$ (recall that we are dealing with real spaces). In order to obtain the other estimate, let $T_{\mathbb{C}}$ denote the complexification of $T$. If $T$ is a bounded band preserving operator, for the spectral radius $r\left(T_{\mathbb{C}}\right)$ of $T_{\mathbb{C}}$ we have $r\left(T_{\mathbb{C}}\right)=\left\|T_{\mathbb{C}}\right\|=\|T\|$, cf. [A, Satz 1.8]. Let $\omega$ be the growth bound of $T_{\mathbb{C}}(t)$. By [N2, Prop. A.III.1.1] we have

$$
\|T(t)\|=r\left(T_{\mathbb{C}}(t)\right)=e^{\omega t} .
$$

The desired estimate now follows from the inequality $T(t) \leqslant\|T(t)\| I[\mathrm{~A}$, Satz 1.8]. ////

Lemma 1.4. If $T(t)$ is a multiplication semigroup on $E$, then $D(A)$ is an ideal.

Proof: Suppose $0 \leqslant|x| \leqslant|y|$ with $y \in D(A)$. Then for $t, s>0$ we have by Lemma 1.1(ii)

$$
\begin{aligned}
\left|\frac{1}{t}(T(t) x-x)-\frac{1}{s}(T(s) x-x)\right| & =\left|\frac{1}{t}(T(t)-I)-\frac{1}{s}(T(s)-I)\right||x| \\
& \leqslant\left|\frac{1}{t}(T(t)-I)-\frac{1}{s}(T(s)-I)\right||y| \\
& =\left|\frac{1}{t}(T(t) y-y)-\frac{1}{s}(T(s) y-y)\right| .
\end{aligned}
$$

Therefore

$$
\left\|\frac{1}{t}(T(t) x-x)-\frac{1}{s}(T(s) x-x)\right\| \leqslant\left\|\frac{1}{t}(T(t) y-y)-\frac{1}{s}(T(s) y-y)\right\| .
$$

As $t, s \downarrow 0$, the right hand side converges to zero. Therefore the net $\left(t^{-1}(T(t) x-x)\right)_{t \downarrow 0}$ is Cauchy, so $x \in D(A)$. $\quad / / / /$

Let $\omega$ be as in Lemma 1.3. It is easy to see that the resolvent $R(\lambda, A)=(\lambda-A)^{-1}$ is band preserving for $\lambda>\omega$ : this follows from

$$
0 \leqslant R(\lambda, A) x=\int_{0}^{\infty} e^{-\lambda t} T(t) x d t \leqslant \frac{1}{\lambda-\omega} x, \quad x \geqslant 0
$$

The above lemma shows that the range of $R(\lambda, A)$ is an ideal. Unless $E$ is Dedekind complete, in general it is not true that the range of an arbitrary band preserving operator $M$ is an ideal. A simple counterexample is given by $E=C[-1,1], M f(x)=x f(x)$. The function $g(x)=0$, $x \leqslant 0$ and $g(x)=x, x \geqslant 0$ belongs to the ideal generated range $(M)$ but not to range $(M)$ itself. Cf. also Remark 2.12. 
Let $\mathcal{A}$ denote the class of all densely defined linear operators on $E$ with the properties that (i) $D(A)$ is an ideal, (ii) $A$ is band preserving, and (iii) $A$ is bounded from above, i.e, $\omega-A \geqslant 0$ for some $\omega \in \mathbb{R}$. The set $\mathcal{A}$ is partially ordered in a natural way by saying that $A \subset B$ if $B$ is an extension of $A$.

The next theorem describes the structure of $\mathcal{A}$.

Theorem 1.5. For a linear operator $A$ on a Banach lattice $E$ the following assertions are equivalent:

(i) $A$ is the generator of a multiplication semigroup;

(ii) $A$ is a maximal element of $\mathcal{A}$;

(iii) $A$ is a closed element of $\mathcal{A}$.

Moreover, each $A \in \mathcal{A}$ is closable and $\bar{A}$ generates a multiplication semigroup.

Proof: (Step 1) We start with (i) $\Rightarrow$ (iii). As a generator, $A$ is closed. By Lemma $1.4, D(A)$ is an ideal in $E$. If $x \in D(A)$, then $A x=\lim _{t \downarrow 0} t^{-1}(T(t) x-x) \in \overline{E_{x}} \subset B_{x}$. Moreover, for $0 \leqslant x \in D(A)$ we get

$$
\frac{1}{t}(T(t) x-x) \leqslant \frac{1}{t}\left(e^{\omega t}-1\right) x
$$

and letting $t \downarrow 0$ we obtain $A x \leqslant \omega x$. This proves that $A \in \mathcal{A}$.

(Step 2) We prove: If $A \in \mathcal{A}$, then $A$ is closable and $\bar{A}$ generates a multiplication semigroup on $E$. Before turning to the proof, we note that (iii) $\Rightarrow$ (i) follows from this.

Without loss of generality assume that $A \leqslant 0$. Let $0 \leqslant u \in D(A), v:=-A u \geqslant 0$. There exists a band preserving linear operator $M \in L\left(E_{u+v}\right)$ such that $M(u+v)=u$. In fact, representing $E_{u+v}$ as a space $C(K)$ such that $u+v$ corresponds to the function $1_{K}$, we may take $M$ to be multiplication with the function corresponding to $u$. The operator $S: E_{u+v} \rightarrow E_{u+v}$ given by $S f:=(I-A) M f$ is well-defined: for $M$ maps $E_{u+v}$ into $E_{u}$, and $E_{u} \subset D(A)$ since $D(A)$ is an ideal. Also, $S$ is band preserving. Since $S(u+v)=u+v$, it follows from $\left[\mathrm{AB}\right.$, Thm. 8.14] that $S=I$ on $E_{u+v}$. This shows that $E_{u+v} \subset \operatorname{range}(I-A)$. In particular $u \in \operatorname{range}(I-A)$ and consequently range $(I-A)$ is dense. Next we prove that $A$ is dispersive. Let $f \in D(A), g:=f-A f$. We must prove that $\left\|g^{+}\right\| \geqslant\left\|f^{+}\right\|$. Put $u:=|f|$ and $v:=-A u \geqslant 0$. Let $M$ be as above and define $R \in L\left(E_{u}\right)$ by $R h:=M(I-A) h$. Then $R$ is well-defined and band preserving. Since $R u=u$ it follows that $R=I$ on $E_{u}$. In particular $M g=M(I-A) f=f$. Hence $M g^{+}=f^{+}$. Since $0 \leqslant M \leqslant I$, it follows that $\left\|g^{+}\right\| \geqslant\left\|f^{+}\right\|$, so $A$ is dispersive. By [N2, Cor. C.II.1.3], $\bar{A}$ generates a positive semigroup $T(t)$. In particular $R(\lambda, \bar{A}) \geqslant 0$ for $\lambda$ sufficiently large. It remains to prove that $T(t)$ is a multiplication semigroup. Since $\lambda-\bar{A} \geqslant \lambda$ for all $\lambda>0$, for all $0 \leqslant x \in D(\bar{A})$ and $\lambda$ large enough we have $0 \leqslant \lambda R(\lambda, \bar{A}) x \leqslant R(\lambda, \bar{A})(\lambda-\bar{A}) x=x$. Since $D(\bar{A})$ is dense, it follows that $0 \leqslant R(\lambda, \bar{A}) \leqslant \lambda^{-1} I$, so $R(\lambda, \bar{A})$ is band preserving. By the exponential formula [P, Thm. $1.8 .3]$

$$
T(t) x=\lim _{k \rightarrow \infty}\left(\frac{k}{t} R\left(\frac{k}{t}, \bar{A}\right)\right)^{k} x
$$

we find that $T(t)$ is a multiplication semigroup.

(Step 3) We prove (i) $\Leftrightarrow($ ii). Suppose $A$ generates a multiplication semigroup. By Step $1, A \in \mathcal{A}$. Suppose some $\tilde{B} \in \mathcal{A}$ extends $A$. By Step 2, some extension $B$ of $\tilde{B}$ generates a multiplication semigroup. Then $B$ is an extension of $A$ as well, and both $A$ and $B$ are generators. By a simple standard argument this implies that $A=B$. This shows that $A$ is maximal in $\mathcal{A}$. Conversely, suppose $A$ is maximal in $\mathcal{A}$. By Step $2, \bar{A}$ generates a multiplication semigroup and by Step $1, \bar{A} \in \mathcal{A}$. By the maximality of $A$ we must have $A=\bar{A}$. $\quad / / / /$

Since the intersection of two dense ideals is a dense ideal, from Theorem 1.5 we have: 
Corollary 1.6. Suppose $A$ and $B$ generate multiplication semigroups. Then $A+B$ with $D(A+B)=D(A) \cap D(B)$ is closable and its closure generates a multiplication semigroup. Moreover, $A$ and $B$ commute.

Proof: The first part follows directly from Theorem 1.5. We will prove that $A$ and $B$ commute. Since $R(\lambda, A) R(\lambda, B)=R(\lambda, B) R(\lambda, A)$ we have $D(B A)=D(A B)$ and by taking inverses we obtain $A B=B A$. $\quad / / / /$

\section{Proof of Theorems 0.2 and 0.3}

We will now proceed with the proof of Theorem 0.2. Let us outline the basic idea. We represent $E$ 'locally', i.e. on closed principal ideals, as a space of continuous $\overline{\mathbb{R}}$ valued functions, $\overline{\mathbb{R}}$ denoting the two-point compactification of $\mathbb{R}$, and show that the part of $A$ in such an ideal can be represented as multiplication with some continuous function $g$. Then we extend $A$ as multiplication with $g$.

We will use the representation theory for Banach lattices with quasi-interior points, developed by Davies [D], Lotz [L] and Schaefer [S1]; see also [N1]. Recall that $u$ is a quasi-interior point if the ideal $E_{u}$ is dense in $E$.

Theorem 2.1 [L]. Suppose $E$ is a Banach lattice with quasi-interior point $u>0$. There exists a compact Hausdorff space $K_{u}$ such that $E$ is vector lattice isomorphic to a space $\hat{E}$ of continuous $\overline{\mathbb{R}}$-valued functions on $K_{u}$. Moreover, each $\hat{x} \in \hat{E}$ is finite on an open dense subset of $K_{u}$. The space $\hat{E}$ contains $C\left(K_{u}\right)$ as a dense ideal and $u$ can be identified with the constant one function.

From now on we will identify $\hat{E}$ with $E$.

$E$ is in fact an 'ideal' in the space of all continuous functions $f: K_{u} \rightarrow \overline{\mathbb{R}}$ in the following sense: if $x \in E$ and $f: K_{u} \rightarrow \overline{\mathbb{R}}$ satisfies $0 \leqslant|f(s)| \leqslant|x(s)|$ for all $s \in K_{u}$, then $f$ represents an element $f \in E$. We will refer to this as the ideal property.

Following [N1], call a subset $N \subset K_{u} E$-null if the ideal $\{f \in E: f(s)=0 \quad \forall s \in N\}$ is norm-dense in $E$. A set $N$ is $E$-null if and only if there exists a $g \geqslant 0$ in $E$ such that $N \subset\{g=\infty\}$. If $N$ is $E$-null, then $K_{u}=\beta\left(K_{u} \backslash N\right)$, the Stone-Čech compactification of $K_{u} \backslash N$. Moreover, the complement of each null set is dense.

We need one more description of $E$-null sets. To this end we first note that every element $0 \leqslant x^{*} \in E^{*}$, the dual of $E$, can be identified with a positive Borel measure $\mu_{x^{*}} \in\left(C\left(K_{u}\right)\right)^{*}$ by restriction. A subset $N \subset K_{u}$ is $E$-null if and only if $\mu_{x^{*}}(N)=0$ for all $0 \leqslant x^{*} \in E^{*}$. This implies that the union of countably many $E$-null sets is $E$-null. The proofs can be found in [N1].

Lemma 2.2. Suppose $A$ is a positive band preserving operator on a Banach lattice $E$ with $D(A)$ a Riesz subspace. If $D(A)$ contains a countable subset $\left(f_{n}\right) \geqslant 0$ which generates a dense ideal in $E$, then $E$ has a quasi-interior point $u>0$ and there is a continuous function $0 \leqslant g_{u}: K_{u} \rightarrow \overline{\mathbb{R}}$ such that $A f=g_{u} f$ holds for all $f \in D(A)$.

Proof: For $u$ one can take $\sum_{n} 2^{-n}\left\|f_{n}\right\|^{-1} f_{n}$. Let $K_{u}$ denote the representation space of $E$. Set $N:=\cap_{n}\left\{f_{n}=0\right\}$. Then $N$ is an $E$-null set. Indeed, this follows from the definition of $E$-null and the fact that $\left(f_{n}\right)$ generates a dense ideal in $E$. Put $N_{n}:=\left\{f_{n}=\infty\right\} \cup\left\{A f_{n}=\infty\right\}$. Then each $N_{n}$ is $E$-null and since the countable union of $E$-null sets is $E$-null, so is $M:=N \cup\left(\cup_{n} N_{n}\right)$. Fix $s \notin M$. By the definition of $M$ there is an $n$ such that $0<f_{n}(s)<\infty$ and $0 \leqslant A f_{n}(s)<\infty$. Put

$$
g_{u}(s):=\frac{A f_{n}(s)}{f_{n}(s)}
$$


Note that $0 \leqslant g_{u}(s)<\infty$. If $0 \leqslant h \in D(A)$ is any element satisfying $0<h(s)<\infty$ then we claim that $A h(s)=g_{u}(s) h(s)$ holds. We may assume without loss of generality that $0<h(s)=f_{n}(s)<\infty$. Since $D(A)$ is a Riesz subspace, $h \wedge(1+\varepsilon) f_{n} \in D(A)$ for all $\varepsilon>0$. Note that $\left(h \wedge(1+\varepsilon) f_{n}\right)(\tau)=h(\tau)$ holds on some open neighbourhood of $s$. Hence, since $A$ is band preserving, it follows that $A h(s)=A\left(h \wedge(1+\varepsilon) f_{n}\right)(s)$. By the positivity of $A$ we obtain

$$
A h(s)=A\left(h \wedge(1+\varepsilon) f_{n}\right)(s) \leqslant A\left((1+\varepsilon) f_{n}\right)(s)=(1+\varepsilon) g_{u}(s) f_{n}(s)=(1+\varepsilon) g_{u}(s) h(s) .
$$

Since $\varepsilon$ is arbitrary it follows that $A h(s) \leqslant g_{u}(s) h(s)$. Arguing similarly with $h \vee(1-\varepsilon) f_{n}$ we obtain $A h(s) \geqslant g_{u}(s) h(s)$. This proves the claim. Taking for $h$ any $f_{m}$ with $f_{m}(s)>0$ it follows that $g_{u}(s)$ does not depend on the choice of $n$. If $0 \leqslant f \in D(A)$ is arbitrary, let $N_{f}:=\{f=\infty\}$. We will show that $A f=g_{u} f$ holds outside the $E$-null set $N_{f} \cup M$. If $f(s)>0$ this follows from the claim. If $f(s)=0$ choose $m$ such that $f_{m}(s)>0$ and note that $A f(s)=A\left(f+f_{m}\right)(s)-A f_{m}(s)=g_{u}(s)\left(f+f_{m}\right)(s)-g_{u}(s) f_{m}(s)=g_{u}(s) f(s)$. The proof is completed by the observation that $K_{u} \backslash F$ is dense in $K_{u}$ for every $E$-null set $F \subset K_{u}$. ////

Note that the set $\left\{g_{u}=\infty\right\}$ is null. It is easy to see that if $A$ is a bounded, then $g_{u}$ is bounded as well.

In applying this lemma to the proof of Theorem 0.2 we encounter two difficulties. First, if $0 \leqslant u \in D(A)$, then $A u$ need not a priori belong to $\overline{E_{u}}$ since $A$ is only assumed to be band preserving. Therefore one has to care about the construction of certain ideals on which $A$ can be handled well. Secondly, if $0 \leqslant x \leqslant y$ with $y \in D(A)$ and if $A$ is represented as multiplication with some function $g$, then we have $A y=g y$. We would like to apply the ideal property to the function $g x$ and then define $A x:=g x$. The problem is that the function $g x$ need not a priori be continuous or even well-defined at points $s$ where $g(s)=\infty$ and $f(s)=0$.

Theorem 2.3. Suppose $A$ is an order bounded band preserving operator whose domain $D(A)$ is a Riesz subspace generating a dense ideal. Then $A$ preserves closed ideals. Moreover, $A$ admits a unique extension to a positive operator $\tilde{A}$, preserving closed ideals, whose domain $D(\tilde{A})$ is the ideal generated by $D(A)$.

Proof: By Lemma 1.1 without loss of generality we may assume that $A$ is positive.

(Step 1). In this step we prove the theorem for the special case that $D(A)$ contains a countable subset $\left(f_{n}\right) \geqslant 0$ which generates a dense ideal in $E$. Fixing any quasi-interior point $u>0$, by Lemma 2.2 there is a function $g: K_{u} \rightarrow \overline{\mathbb{R}}$ such that $A y=g y$ for all $y \in D(A)$. Moreover, $N_{g}:=\{g=\infty\}$ is a null set. Let $J$ denote the ideal generated by $D(A)$ and let $0 \leqslant x \in J$ be arbitrary. Choose $f \in D(A)$ such that $0 \leqslant x \leqslant f$.

First we claim that $A f \in \overline{E_{f}}$. To this end, let $N_{f}=\{f=\infty\}$. Define $\phi_{n}:=A f \wedge n f$. Then $\phi_{n} \in E_{f}$ and $\phi_{n}(s) \uparrow A f(s)$ for all $s \in K_{u} \backslash\left(N_{g} \cup N_{f}\right)$. Fix $0 \leqslant x^{*} \in E^{*}$ arbitrary. Identifying $x^{*}$ with a positive Borel measure $\mu_{x^{*}} \in C\left(K_{u}\right)^{*}$, we see that $0 \leqslant \phi_{n} \uparrow$ Af $\mu$-almost everywhere. Since $A f \in L^{1}(\mu)$, the dominated convergence theorem implies that $\phi_{n} \rightarrow A f$ weakly. The convergence being monotone, Dini's theorem implies that $\phi_{n} \rightarrow A f$ in $E$-norm. This proves the claim.

By Lemma 2.2, the part of $A$ in $\overline{E_{f}}$ is represented as multiplication with a function $g_{f}$. Since $1_{K_{f}}=f \leqslant f+x \leqslant 2 f$, the function $g_{f}(f+x)$ is a well-defined continuous $\overline{\mathbb{R}}$ valued function on $K_{f}$. The ideal property, applied to $0 \leqslant g_{f}(f+x) \leqslant 2 A f$ implies that $g_{f}(f+x)$ represents an element $z \in \overline{E_{f}}$. We now define $\tilde{A}(f+x):=z$ and $\tilde{A} x:=z-A f$. Clearly $A x=g_{f} x$ outside some null set. Therefore $\tilde{A}$ extends $A$. In this way we obtain a positive operator $\tilde{A}$ on $\overline{E_{f}}$ with domain $D(\tilde{A})=E_{f}$. Clearly $\tilde{A}$ preserves bands. Therefore, by an argument as above or by Theorem 1.5 and the remark after Lemma 1.2, $\tilde{A}$ preserves closed 
ideals.

We will show that $\tilde{A}$ is well-defined, i.e. does not depend on the choice of $f$. Suppose $0 \leqslant x \leqslant f_{i}$ with $f_{i} \in D(A), i=1,2$. After replacing $f_{1}$ by $f_{1} \wedge f_{2}$ we may assume that $0 \leqslant x \leqslant f_{1} \leqslant f_{2}$. We obtain two extensions $\tilde{A}_{1}$ and $\tilde{A}_{2}$ of $A$, both preserving closed ideals, which are simultaneously defined on the ideal $E_{f_{1}}$. But on $\overline{E_{f_{1}}}$ each $\tilde{A}_{i}$ can be represented as multiplication with continuous $\mathbb{R}$-valued function $g_{i}$. Since $f_{1}$ corresponds to the constant one function on $K_{f_{1}}$, we have $g_{i}=\tilde{A}_{i} f_{1}=A f_{1}$. This proves that $\tilde{A}_{1} x=\tilde{A}_{2} x$.

(Step 2). Now let $E$ be arbitrary and let $J$ be the ideal generated by $D(A)$. Fix $0 \leqslant y \in J$ arbitrary and choose $0 \leqslant x \in D(A)$ such that $0 \leqslant y \leqslant x$. Define the countable sets $S_{n} \subset E_{+}$ and $T_{n} \subset E_{+}$as follows. Put $S_{0}:=\{x\}, T_{0}:=\{A x\}$. Suppose the sets $S_{0}, \ldots, S_{n-1}$ and $T_{0}, \ldots, T_{n-1}$ have been chosen. Since $J$ is dense, for each $y \in T_{n-1}$ there is a sequence $0 \leqslant$ $\left(x_{k}(y)\right) \subset J$ converging to $y$. For each $k$ choose $z_{k}(y) \in D(A)$ such that $0 \leqslant x_{k}(y) \leqslant z_{k}(y)$. Put $S_{n}:=\left\{z_{k}(y): k \in \mathbb{N}, y \in T_{n-1}\right\}$. Note that $S_{n}$ is a countable subset of $D(A)$. Put $T_{n}:=\left\{A y: y \in S_{n}\right\}$. Next we put $S:=\cup_{n} S_{n}$ and $T:=\cup_{n} T_{n}$. Let $F$ be the closed ideal generated by $S$ in $E$. Note that by construction we have $T \subset F$. Moreover, the part $A_{F}$ of $A$ in $F$ is positive and band preserving considered as an operator in $F$, and its domain $D\left(A_{F}\right)$ is a Riesz subspace of $F$ ( since $A|x|=|A x| \in F$ for all $x \in D\left(A_{F}\right)$ ) containing $S$. But the countable set $S \subset D\left(A_{F}\right)$ generates a dense ideal in $F$. Hence by Step 1, $A_{F}$ extends to a positive operator $\tilde{A}_{F}$ on the ideal generated by $D\left(A_{F}\right)$ in $F$ which preserves closed ideals. We define the extension of $A$ by $\tilde{A} y:=A_{F} y$.

It remains to show that $\tilde{A}$ is well-defined, i.e. that the extensions $A_{F}$ glue together to a well-defined linear operator. This can be done as in Step 1. ////

Combining this with Theorem 1.5 gives:

Corollary 2.4. Suppose $A$ is a positive band preserving operator with $D_{\tilde{A}}(A)$ a Riesz subspace which generates a dense ideal. Then $-A$ admits a unique extension $-\tilde{A}$ to a generator of a multiplication semigroup $\tilde{T}(t)$.

Corollary 2.5. Suppose $E$ has a quasi-interior point $u>0$. Then for all $v<0$ there is a unique multiplication semigroup on $E$, with generator $A_{v}$, such that $u \in D\left(A_{v}\right)$ and $A_{v} u=v$.

Corollary 2.5 can be derived directly from Theorem 1.5 as follows. Choose a positive band preserving operator $M$ such that $M(u-v)=u$; this is possible since $0 \leqslant u \leqslant u-v$. Then $M$ is easily seen to be injective. Define $D(A):=M E$ and $A M x:=x-M x$. Then $A$ satisfies the hypotheses of Theorem 1.5. This proofs is interesting as it leads to a representation-free proof of the following consequence of Corollary 2.5 [Ne]: Suppose $E$ is a Banach lattice with quasi-interior point. If every $C_{0}$-semigroup on $E$ is uniformly continuous, then $E$ has the Grothendieck property and there is a compact Hausdorff space $K$ such that $E$ is Banach lattice isomorphic to $C(K)$. Since every $C(K)$-space has the Dunford-Pettis property, this is a partial converse of Lotz's theorem that every $C_{0}$-semigroup on a Banach space with the Grothendieck- and the Dunford-Pettis property is uniformly continuous. This converse fails if $E$ has only a weak order unit; see [Le].

Corollary 2.6. Suppose $A$ is an order bounded operator which preserves closed ideals with $D(A)$ a Riesz subspace. Then $A$ admits a unique extension to an operator preserving closed ideals whose domains is the ideal generated by $D(A)$ in $E$.

Just apply Theorem 2.3 to the closed ideal generated by $D(A)$.

Corollary 2.7. If two generators $A, B$ of multiplication semigroups agree on a Riesz subspace $F$ of $D(A) \cap D(B)$ which generates a dense ideal, then $A=B$. 
In particular it follows that $D(A)=D(B)$. With a little more care one can show that Corollary 2.7 is true even if $F$ is only a linear subspace of $D(A) \cap D(B)$ generating a dense ideal.

Corollary 2.8. Suppose $F \subset E$ is a closed sublattice generating a dense ideal. If $T \in \mathcal{L}(F)$ is a band preserving operator on $F$, then there is a unique extension $\tilde{T}$ to a band preserving operator on $E$.

Proof: By Lemmas 1.1 and 1.2 we may assume $0 \leqslant T \leqslant I$. By Theorem 2.3 there is an extension $\tilde{T}$ to a linear band preserving operator $0 \leqslant \tilde{T} \leqslant I$ whose domain is the dense ideal generated by $F$ in $E$. But the inequalities implies that $\tilde{T}$ is bounded; its extension to a bounded operator on $E$ again satisfies $0 \leqslant T \leqslant I$. ////

Remark 2.9. (i) This result is related to the following theorem due to Luxemburg and Schep [M, Thm. 1.5.15]: If $F$ is a majorizing Riesz subspace of the Dedekind complete Riesz space $E$ and if $T: F \rightarrow E$ is a lattice homomorphism, then $T$ extends to a lattice homomorphism $E \rightarrow E$. Recall that a Riesz subspace of $E$ is called majorizing if the ideal generated by it is E.

(ii) The following example shows that the word 'ideal' in 2.8 cannot be replaced by 'band'. Let $E=C[-1,1], F=\{f \in E: f(0)=0\}$,

$$
T f(s)= \begin{cases}0, & s \leqslant 0 \\ f(s), & s>0\end{cases}
$$

Since every band preserving operator on $C[-1,1]$ is multiplication with a continuous function $g$ (e.g. by Lemma 2.2), $\tilde{T}$ can only be multiplication with a function $g$ which equals zero on $[-1,0)$ and one on $(0,1]$; such $g$ fails to be continuous.

Note that this is a counterexample to [M, Thm. 3.1.19(ii)].

The following examples show that Theorem 2.3 is sharp.

Example 2.10. (i) Let $E=L^{\infty}[0,1]$, define $D(A):=\left\{f \in E: x^{-1} f(x) \in E\right\}$ and put $A f(x)=x^{-1} f(x), f \in D(A)$. Then $D(A)$ is an order dense ideal in $E, A$ is positive and band preserving, but not preserving closed ideals. This shows that Theorem 2.3 fails if the ideal (generated by) $D(A)$ is not norm-dense, even if it is order dense.

(ii) Let $E=C[0,1]$, define $D(A)$ to be the set of all polynomials in $E$, and for $f \in D(A)$

put $A f:=\int_{0}^{1} f(x) d x$. Then $A$ is positive and band preserving and $D(A)$ is dense, but $A$ does not preserve closed ideals. This shows that Theorem 2.3 fails if $D(A)$ is not a Riesz subspace. By taking for $D(A)$ the set of functions of the form $f(x)=a x+b$ with $a, b \in \mathbb{R}$ we see that Theorem 2.3 even fails in case $D(A)$ is a Riesz space in its own right.

(iii) Let $E=L^{\infty}[0,1]$, let $D(A)$ be the subspace of all bounded, piecewise linear functions consisting of finitely many pieces. For $f \in D(A)$ put $A f:=d f / d x$. Then $D(A)$ is a Riesz subspace of $E$ generating a dense ideal, $A$ preserves bands but not closed ideals. This shows that Theorem 2.3 fails if $A$ is not order bounded.

We now come to the proof of Theorem 0.3.

Theorem 2.11. Let $A$ be a linear operator on $E$ whose domain generates a dense ideal. Then $A$ generates a multiplication semigroup if and only if there is a $\lambda \in \varrho(A)$ such that $R(\lambda, A)$ is positive and band preserving.

Proof: First, $|R(\lambda, A) x|=R(\lambda, A)|x|$ shows that $D(A)=R(\lambda, A) E$ is a sublattice of $E$. We claim that $\lambda-A$ is positive and closed ideal preserving. Suppose $0 \leqslant x=R(\lambda, A) y$. We must 
prove that $y \geqslant 0$. Indeed, since $R(\lambda, A) y=x=x^{+}=R(\lambda, A) y^{+}$, the injectivity of $R(\lambda, A)$ shows that $y=y^{+}$, proving positivity. Now fix $u \geqslant 0$ arbitrary and represent the restriction of $R(\lambda, A)$ to $\overline{E_{u}}$ as multiplication with $g_{u} \in C\left(K_{u}\right)$. We claim that $\{g>0\}$ is open dense in $K_{u}$. Indeed, if not, then one finds a non-zero Urysohn function $f \in C\left(K_{u}\right)$ supported in $\{g=0\}$. But then $R(\lambda, A) f=g_{u} f=0$, a contradiction to the injectivity of $R(\lambda, A)$. For all $s \notin\{g=0\}$ we have

$$
(u \wedge n R(\lambda, A) u)(s) \uparrow_{n} u(s) .
$$

Since the set of these $s$ is open dense, it follows that $u \wedge n R(\lambda, A) u \uparrow u$, so $u$ belongs to the band generated by $R(\lambda, A) u$. In other words, $\lambda-A$ is band preserving.

Putting together what we have proved so far, an application of Theorem 2.3 shows that $A$ preserves closed ideals and admits a unique extension to a closed ideal preserving operator $\tilde{A}$ whose domain is the ideal generated by $D(A)$ such that $\lambda-\tilde{A} \geqslant 0$.

We claim that $\lambda-\tilde{A}$ is injective. Indeed, suppose $(\lambda-\tilde{A}) u=0$ for some $u \in D(\tilde{A})$. Choose $|u| \leqslant v \in D(A)$. Since both $\lambda-A$ and $\lambda-\tilde{A}$ preserve closed ideals, we may apply Lemma 2.2 to their parts in $\overline{E_{v}}$ and represent them as multiplication with functions $g_{v}$ and $h_{v}$ respectively. Since $1_{K_{v}}=v \in D(A) \subset D(\tilde{A})$, necessarily $g_{v}=h_{v}$. As above, $\left\{g_{v}>0\right\}=\left\{h_{v}>0\right\}$ is open dense; but if $u \neq 0$, then $\left\{h_{v}=0\right\}$ is non-empty and open because of $(\lambda-\tilde{A}) u=0$. Therefore $u=0$.

But $\lambda-A$ is $1-1$ and onto whereas its extension $\lambda-\tilde{A}$ is still $1-1$. Therefore $A=\tilde{A}$ and consequently $D(A)$ is an ideal. Also, $A$ is closed, since $\lambda-A$ is the inverse of a bounded operator. Therefore, by Theorem 1.5, $A$ generates multiplication semigroup. ////

This is a remarkable result in that it gives a generation criterium in terms of one single resolvent operator. Also note that the hypotheses are almost exclusively lattice-theoretic, the only Banach space hypothesis being that the ideal generated by $D(A)$ be dense. A similar situation occurs in the following result of Arendt, Chernoff and Kato [N2, Thm. B.II.1.8]: If $A$ is a densely defined operator on a $C(K)$-space $E$ such that there exists a sequence $\varrho(A) \ni \lambda_{n} \rightarrow \infty$ with $R\left(\lambda_{n}, A\right) \geqslant 0$ for all $n$, then $A$ generates a positive semigroup on $E$.

Remark 2.12. Theorem 2.11 can be reformulated as follows: If $0 \leqslant M$ is a bounded band preserving operator on $E$ whose range generates a dense ideal, then its range is an ideal. Indeed, the hypothesis on $M$ guarantees that $M$ is 1-1 and we can apply 2.11 to its inverse. One can show that the positivity assumption on $M$ is actually redundant.

There are many positive band preserving operators whose domain fails to be a Riesz subspace. A very general example of this situation is the following, which arises e.g. in the context of differential operators.

Example 2.13. Let $A$ be the generator of a multiplication semigroup on a Banach lattice $E$. Since generators are closed, $D(A)$ is a Banach space in the graph norm $\|\cdot\|_{A}$. Let $\phi$ be any bounded linear functional on $\left(D(A),\|\cdot\|_{A}\right)$ which is unbounded with respect to the $E$-norm. For instance one might take $E=l^{1}, A x_{n}:=-n x_{n}$ with maximal domain $D(A)$ and $\phi(x):=\sum_{n=1}^{\infty} n x_{n}$. Let $N_{\phi}$ denote the kernel of $\phi$. Then

$$
\begin{aligned}
& D\left(A_{\phi}\right):=N_{\phi} ; \\
& A_{\phi} x:=A x, \quad x \in D\left(A_{\phi}\right)
\end{aligned}
$$

is a closed densely defined operator. Closedness is immediate from the continuity of $\phi$ with respect to the graph norm and denseness of $D\left(A_{\phi}\right)$ follows easily from the denseness of $D(A)$ in combination with the unboundedness of $\phi$ with respect to the $E$-norm. Moreover $A_{\phi}$ preserves closed ideals since $A$ does and also $A_{\phi}$ is bounded from above. $A_{\phi}$ is not a generator, since 
from $A_{\phi} \subset A$ we would have that $A_{\phi}=A$ which is not the case since $\phi \neq 0$ implies that the inclusion $D\left(A_{\phi}\right) \subset D(A)$ is proper.

Many of the results in this paper carry over to the complex case. For example, suppose $E$ is a complex Banach lattice and $A=A_{0}+i A_{1}$ is a linear operator in $E$ with both $A_{j}$ real. If (i) $A$ is order bounded and band preserving, (ii) $D(A)$ is a dense ideal and (iii) the real part $A_{0}$ is bounded from above, then $A$ is closable and $\bar{A}$ generates a multiplication semigroup. With this in mind we have the following illustration of Example 2.13. Let $E=L^{2}[0,1]$ and define operators $A_{i}, i=0,1,2$ by

$$
\begin{aligned}
& D\left(A_{0}\right)=\left\{f \in W^{1,2}[0,1]: f(0)=f(1)\right\}, \\
& D\left(A_{1}\right)=\left\{f \in W^{1,2}[0,1]: f(1)=0\right\}, \\
& D\left(A_{2}\right)=D\left(A_{0}\right) \cap D\left(A_{1}\right),
\end{aligned}
$$

with $A_{i} f=f^{\prime}$. After taking Fourier transforms we obtain operators $\tilde{A}_{i}$ on $l^{2}(\mathbb{Z})$. It is easy to see that both $\tilde{A}_{0}$ and $\tilde{A}_{1}$ are generators of strongly continuous semigroups, of which the one generated by $\tilde{A}_{0}$ is a multiplication semigroup. Moreover, in the terminology of 2.13 we have $A_{2}=\left(A_{0}\right)_{\phi}$, where $\phi f=f(1)$ so $\tilde{A}_{2}$ fails to generate a semigroup. Note that $\tilde{A}_{2}$ admits at least two different extensions to a generator, but only one of them generates a multiplication semigroup.

\section{The adjoint of a multiplication semigroup}

In this final section we are concerned with the duality theory of multiplication semigroups.

If $T(t)$ is a $C_{0}$-semigroup on a Banach space $X$, then for each $t \geqslant 0$ let $T^{*}(t)$ be the adjoint of the operator $T(t)$. The operators $T^{*}(t)$ form a semigroup on $X^{*}$ which however need not be strongly continuous. Put

$$
X^{\odot}:=\left\{x^{*} \in X^{*}: \lim _{t \downarrow 0}\left\|T^{*}(t) x^{*}-x^{*}\right\|=0\right\} .
$$

Then $X^{\odot}$ is a weak*-dense, norm-closed subspace of $X^{*}$. In fact we have $X^{\odot}=\overline{D\left(A^{*}\right)}$, where $A^{*}$ is the adjoint of $A$. The restrictions $T^{\odot}(t)$ of $T^{*}(t)$ to $X^{\odot}$ define a $C_{0}$-semigroup on $X^{\odot}$ whose generator $A^{\odot}$ is precisely the part of $A^{*}$ in $X^{\odot}$. For a proof of these facts see [BB]. By repeating this construction one defines $X^{\odot \odot}:=\left(X^{\odot}\right) \odot$ and similarly $T^{\odot \odot}(t)$ and $A^{\odot \odot}$. The

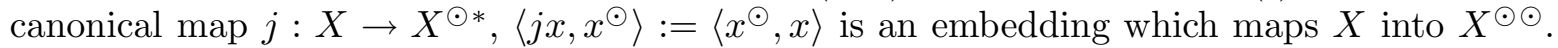
Thus we can identify $X$ with a closed subspace of $X \odot \odot$. In doing so, $T^{\odot \odot}(t)$ is an extension of $T(t)$ and the same remark applies to $A \odot \odot$. For the proofs see [HPh]. A well-known theorem of Phillips $[\mathrm{Ph}]$ states that if $X$ is a reflexive Banach space, then $X^{\odot}=X^{*}$.

Theorem 3.1. Let $T(t)$ be a multiplication semigroup on a Banach lattice $E$.

(i) $E^{\odot}$ is an ideal in $E^{*}$ and $T^{\odot}(t)$ is a multiplication semigroup on $E^{\odot}$.

(ii) If $E^{*}$ has order continuous norm, then $E^{\odot}=E^{*}$.

Proof: (i) Suppose $0 \leqslant\left|x^{*}\right| \leqslant\left|y^{*}\right|$ holds with $y^{*} \in E^{\odot}$. Since each $T^{*}(t)$ is a band preserving operator by Lemma 1.2(d), by Lemma 1.1(ii) we have

$$
\left|T^{*}(t) x^{*}-x^{*}\right|=\left|T^{*}(t)-I\right|\left|x^{*}\right| \leqslant\left|T^{*}(t)-I\right|\left|y^{*}\right|=\left|T^{*}(t) y^{*}-y^{*}\right| .
$$

By the lattice property of the norm, $x^{*} \in E^{\odot}$ and therefore $E^{\odot}$ is an ideal. Once more by Lemma 1.2 , each $T^{\odot}(t)$ is a multiplication operator. This proves the first assertion.

(ii) First we claim that $A^{*}$ is band preserving. Fix $x^{*} \in D\left(A^{*}\right)$ and let $\pi$ be the band 
projection onto the band generated by $x^{*}$. It follows from Lemma 1.2 that $R\left(\lambda, A^{*}\right)=R(\lambda, A)^{*}$ is band preserving, so

$$
R\left(\lambda, A^{*}\right)\left(\lambda-A^{*}\right) x^{*}=\pi R\left(\lambda, A^{*}\right)\left(\lambda-A^{*}\right) x^{*}=R\left(\lambda, A^{*}\right) \pi\left(\lambda-A^{*}\right) x^{*} .
$$

It follows that $R\left(\lambda, A^{*}\right)(1-\pi)\left(\lambda-A^{*}\right) x^{*}=0$ and therefore $(1-\pi)\left(\lambda-A^{*}\right) x^{*}=0$ by the injectivity of $R\left(\lambda, A^{*}\right)$. This proves the claim. Since $E^{*}$ has order continuous norm, $E^{\odot}$ is a band (since it is a closed ideal by the above). Since $D\left(A^{*}\right) \subset E^{\odot}$, for $x^{*} \in D\left(A^{*}\right)$ it follows that $A^{*} x^{*} \in B_{x^{*}} \subset E^{\odot}$. Since $A^{\odot}$ is the part of $A^{*}$ in $E^{\odot}$ it follows that $A^{\odot}=A^{*}$ and hence $E^{\odot}=E^{*} . \quad / / /$

Recall that every reflexive Banach lattice has order continuous norm.

If $E^{*}$ does not have order continuous norm it can happen that $E^{\odot}$ is a proper subspace of $E^{*}$, as is shown by the example $E=L^{1}[0,1], A f(x)=-x^{-1} f(x)$ with $D(A)$ maximal. However for arbitrary $E$ one can show that the adjoint of a multiplication semigroup is always strongly continuous for $t>0$.

Let $T(t)$ be a $C_{0}$-semigroup on a Banach space $X$. If the canonical map $j: X \rightarrow X^{\odot *}$ maps $X$ onto $X \odot \odot$ then the Banach space $X$ is called $\odot$-reflexive with respect to $T(t)$. This is the case if and only if the resolvent $R(\lambda, A)$ is weakly compact [Pa]. ¿From this it follows easily that each $T(t)$-invariant closed subspace of a $\odot$-reflexive $X$ is $\odot$-reflexive with respect to the restricted semigroup.

Trivially, if $X$ is reflexive, then $X$ is $\odot$-reflexive with respect to any semigroup on $X$. If $E=c_{0}$ or $l^{1}$ then $E$ is $\odot$-reflexive with respect to the multiplication semigroup $T(t)$ defined by $T(t) x_{n}=e^{-n t} x_{n}$, where $x_{n}$ is the $n$th unit vector. Note that both $c_{0}$ and $l^{1}$ are atomic Banach lattices. Recall that $x \in E$ is called an atom if the principal ideal generated by $x$ is one-dimensional and that a Banach lattice is atomic if there exists a maximal orthogonal system $\left\{x_{\alpha}\right\}_{\alpha}$ with each $x_{\alpha}$ an atom. The band $E_{a}$ generated by all atoms of $E$ is called the atomic part of $E$ and is an atomic Banach lattice. Finite-dimensional Banach lattices are atomic. See [S2] for more information.

We will prove that reflexive Banach lattices and atomic Banach lattices with order continuous norm are essentially the only ones which can be $\odot$-reflexive with respect to a multiplication semigroup. This was conjectured by Ben de Pagter (oral communication).

In $[\mathrm{NP}]$ it is shown that whenever a $\sigma$-Dedekind complete Banach lattice $E$ is $\odot$-reflexive with respect to some $C_{0}$-semigroup, then $E$ necessarily has order continuous norm. For convenience of the reader, we give the short proof. First we claim that $E$ does not contain a closed subspace isomorphic to $l^{\infty}$. Suppose the contrary and let $Y$ be a closed subspace of $E$ which is isomorphic to $l^{\infty}$. Since $l^{\infty}$ is complemented in every Banach space containing it as a closed subspace [DU, p. 178], it follows that $Y$ is complemented in $E$. Since $E$ is weakly compactly generated (WCG) by the weak compactness of $R(\lambda, A)$, and since complemented subspaces of WCG spaces are trivially seen to be WCG again, we conclude that $l^{\infty}$ is WCG, a contradiction. In fact, every weakly compact set of $l^{\infty}$ is separable (e.g. note that $l^{\infty}$ embeds into $L^{\infty}[0,1]$ and apply [DU, Thm. VIII.4.13]). This proves the claim. But a $\sigma$-Dedekind complete space without copies of $l^{\infty}$ has order continuous norm [AB, Thm. 14.9].

Lemma 3.2. If $E$ is $\odot$-reflexive with respect to a multiplication semigroup then $E$ has order continuous norm.

Proof: From Theorem 3.1 we know that $E^{\odot \odot}$, being an ideal in the Dedekind complete Banach lattice $E^{\odot *}$, is a Dedekind complete Banach lattice. Clearly $E^{\odot \odot}$ is $\odot$-reflexive with respect to $T^{\odot \odot}(t)$, so by the above remarks $E \odot \odot$ has order continuous norm. Let $0 \leqslant x_{\alpha} \uparrow x$ in $E$. 
Since $j$ is positive we have $0 \leqslant j x_{\alpha} \uparrow \leqslant j x$ and hence $\left(j x_{\alpha}\right)$ is norm convergent to $\sup j x_{\alpha}$. Thus $\left(x_{\alpha}\right)$ is norm convergent as well and its limit must be $x$, which shows that $E$ has order continuous norm. ////

Let $K$ be a subset of a Banach space $X$. In the next lemma we use the standard fact [AB, Thm. 10.17] that if for each $\varepsilon>0$ there exists a weakly compact subset $K_{\varepsilon} \subset X$ such that $K \subset K_{\varepsilon}+\varepsilon U_{X}$, where $U_{X}$ is the closed unit ball of $X$, then $K$ is weakly compact.

Lemma 3.3. If a Banach lattice $E$ is $\odot$-reflexive with respect to a multiplication semigroup $T(t)$, then $T(t)$ is weakly compact for $t>0$.

Proof: Let $\left(x_{n}\right)$ be a bounded sequence in $E$ and let $t>0$ be fixed. By the Eberlein-Shmulyan theorem it suffices to show that the sequence $\left(T(t) x_{n}\right)$ has a weakly convergent subsequence. The closed linear span of $\left(x_{n}\right)$ is contained in some closed principal ideal of $E$, invariant under $T(t)$. Therefore without loss of generality we may assume that $E$ has a quasi-interior point $u>0$. Also we may assume $0 \leqslant T(t) \leqslant I$. Let the generator $A$ be represented on $K_{u}$ as multiplication with a continuous $\overline{\mathbb{R}}$-valued function $g \leqslant 0$. Define the open sets $F_{n}$ by

$$
F_{n}:=\left\{s \in K_{u}:-n<g(s) \leqslant 0\right\}
$$

and let $G_{n}$ be its closure. Since $E$ is Dedekind complete, $K_{u}$ is Stonean and consequently $G_{n}$ is clopen. Define bandprojections $\pi_{n}$ on $E$ by $\pi_{n} x:=\chi_{G_{n}} x$ and denote the corresponding bands by $B_{n}\left(\chi_{G_{n}}\right.$ is the characteristic function of $\left.G_{n}\right)$. The restriction of the semigroup $T(t)$ to each $B_{n}$ is uniformly continuous by construction. Hence $B_{n}^{\odot}=B_{n}^{*}$ and $B_{n}=B_{n}^{\odot \odot}=B_{n}^{* *}$ so each $B_{n}$ is reflexive. Moreover for $t>0$ we have

$$
T(t) U_{E} \subset U_{B_{n}}+e^{-n t} U_{E}
$$

since $0 \leqslant T(t) \leqslant e^{-n t}$ holds on the orthogonal complement of $B_{n}$. By the above remark the weak compactness of $T(t)$ follows. ////

Theorem 3.4. Let $E$ be $\odot$-reflexive with respect to a multiplication semigroup. Then $E$ has order continuous norm, and either $E$ contains an infinite-dimensional reflexive band or $E$ is atomic.

Proof: Suppose there are no infinite-dimensional reflexive bands in $E$. Let $E_{a}$ denote the atomic part of $E$. We will show that $E=E_{a}$. If not, then $E=E_{a} \oplus B$ for some nonempty band $B$. The proof of the previous lemma shows how to find a reflexive band in $B$ which by assumption must be finite-dimensional. But finite-dimensional Banach lattices are atomic, a contradiction to the definition of $E_{a}$. ////

A Banach space $X$ is said to have the Dunford-Pettis property if the square of each weakly compact operator on $X$ is compact. Every $A M$-space and every $A L$-space is Dunford-Pettis [AB]. A Dunford-Pettis space cannot contain a complemented infinite-dimensional reflexive subspace, for then the associated projection $\pi=\pi^{2}$ would be compact.

Corollary 3.5. If $E$ is a Dunford-Pettis lattice which is $\odot$-reflexive with respect to a multiplication semigroup $T(t)$, then $E$ is atomic and $T(t)$ is compact.

Acknowledgement - This paper was written during a half-year visit at the University of Tübingen. I would like to thank the CWI in Amsterdam for giving me the opportunity to visit Tübingen and all members of the faculty in Tübingen for their warm hospitality. Especially I thank Rainer Nagel and Günther Greiner for their many valuable comments on this paper. 
I am also indebted to Ben de Pagter for some interesting discussions and to the referees for their valuable comments and suggesting a slight generalization of Theorem 1.5 along with a simplified proof.

\section{References}

[AB] Aliprantis, C.D., and O. Burkinshaw, "Positive Operators", Pure and Applied Math. 119, Academic Press, 1985

[A] Arendt, W., "Über das Spektrum regulärer Operatoren", Dissertation, Tübingen, 1979

[BB] Butzer, P.L., and H. Berens, "Semigroups of Operators and Approximation", Springer-Verlag, Berlin-Heidelberg-New York, 1967

[D] Davies, E.B., The Choquet theory and representation of ordered Banach spaces, Illinois J. Math. 13 (1969), 176-187

[DU] Diestel, J., and J.J. Uhl, "Vector measures", Math. Surveys nr. 15, Amer. Math. Soc., Providence, R. I., 1977

[HPh] Hille, E., and R.S. Phillips, "Functional Analysis and Semigroups", Amer. Math. Soc. Coll. Publ. 31, Providence, R.I., 1957

[Le] Leung, D., Uniform convergence of operators and Grothendieck spaces with the Dunford-Pettis property, Math. Z. 197 (1988), 21-32.

[L] Lotz, H.P., "Zur Idealstruktur in Banachverbänden", Habilitationsschrift, Tübingen, 1969

[M] Meyer-Nieberg, P., "Banach Lattices", Springer-Verlag, Berlin-Heidelberg-New York (1992). $75-82$

[N1] Nagel, R., A Stone-Weierstrass theorem for Banach lattices, Stud. Math. 47 (1973),

[N2] Nagel, R. (ed.), "One-parameter semigroups of positive operators", Lecture Notes in Math. 1184, Springer-Verlag, Berlin-Heidelberg-New York-Tokyo, 1986

[Ne] Neerven, J.M.A.M. van, A converse of Lotz's theorem on uniformly continuous semigroups, to appear in: Proceedings of the AMS.

[NP] Neerven, J.M.A.M. van, and B. de Pagter, Certain semigroups on Banach function spaces and their adjoints, in: "Semigroup theory and Evolution Equations", Lecture Notes in Pure and Applied Mathematics, Vol. 135, Marcel Dekker Inc., New York-Basel (1991).

[P] Pazy, A., "Semigroups of Linear Operators and Applications to Partial Differential Equations", Springer-Verlag, Berlin-Heidelberg-New York-Tokyo, 1983

[Pa] Pagter, B. de, A characterization of sun-reflexivity, Math. Ann. 283 (1989), 511-518

[Ph] Phillips, R.S., The adjoint semi-group, Pac. J. Math. 5 (1955), 269-283

[S1] Schaefer, H.H., On the representation of Banach lattices by continuous numerical functions, Math. Z. 125 (1972), 215-232

[S2] Schaefer, H.H., "Banach Lattices and Positive Operators", Springer-Verlag, BerlinHeidelberg-New York, 1974 